\title{
SUBACUTE SCLEROSING (INCLUSION BODY) ENCEPHALITIS
}

\author{
BY \\ N. S. CLARK and P. V. BEST \\ From the Departments of Child Health and Pathology, University of Aberdeen
}

(RECEIVED FOR PUBLICATION JANUARY 13, 1964)

The subacute leucoencephalitis of van Bogaert (1945) is now recognized by its characteristic clinical and electroencephalographic pattern as an entity distinct from the many other varieties of cerebral disturbance in childhood grouped under the general title of 'encephalitis". There is a considerable measure of agreement (Foley and Williams, 1953; Poser and Radermecker, 1957; Simpson, 1961) that this condition is identical with the inclusion body encephalitis originally described by Dawson $(1933,1934)$, but its aetiology remains obscure. Although virus infection has long been suspected, most reported studies have failed to provide any clear evidence of association with any known virus. Simpson (1961) has suggested that this form of encephalitis may be a rare manifestation of infection by a virus which usually causes infective hepatitis, but has also pointed out that inclusion bodies may have a biochemical origin and are not necessarily proof of a virus infection.

Four cases of this condition have been recognized in the North-Eastern Region of Scotland (total population approximately 490,000 ) in the years 1952-61, and one other case was referred here for investigation from the Northern Region. These five patients, two of whom were brother and sister, form the subject of the present paper. So far as we are aware, there is only one previously recorded example of the occurrence of this disease in two members of the same family (Lóránd, Nagy, and Tariska, 1962).

\section{Case Reports}

Case 1. A girl aged 8 years was admitted to the Royal Aberdeen Hospital for Sick Children on July 14, 1952. For the previous four months a steady deterioration had been noted in her work at school, and she had been subject to frequent attacks of screaming and weeping for no adequate reason. Soon after these changes were noted, she became clumsy and stumbled frequently and her mother felt that her vision was deteriorating. She had previously been a healthy child and gave no history of obvious physical illness at the time her symptoms were first noticed. On examination she was a dull-looking child with whom it was difficult to maintain contact. Neurological examination was limited by her pcor co-operation. Though there was no alteration of tone or loss of power, her movements were generally clumsy and poorly sustained. No other abnormal signs were detected. The cer zbrospinal fluid protein was $25 \mathrm{mg}$./100 ml., cells 1 /c.mm., colloidal gold $54.432,200$, W.R. negative. Electroencephalography showed generalized complexes consisting of two or three high voltage slow waves recurring every 5 to 7 seconds throughout the recording. During six weeks in the ward there was little change in her condition; the general poverty of movement, speech, and emotional expression continued. While she could name common objects correctly, she was unable to read the simplest words, though before her illness she had been reading well. From time to time she had repeated attacks of petit mal with head-nodding, but no other spontaneous movements were seen. When she attended as an out patient 6 months later, marked deterioration was obvious. She showed frequent irregular athetoid movements, and at times regularly repeated myoclonic jerks of all her limbs. She was incontinent and could no longer walk or feed herself. We did not see her again, but she continued to deteriorate slowly and died at home with a terminal bronchopneumonia on September 27 , 1955 after an illness lasting three and a half years. Necropsy was not performed.

Case 2. A girl aged 11 years was transferred from the Royal Northern Infirmary, Inverness, to this hospital for neurosurgical investigation on September 6, 1954 . Over the past year there had been a steady deterioration in her school work and her behaviour, and for six months she had stumbled readily and shown intermittent irregular jerky movements. On examination she showed, on the left side, a doubtful extensor plantar response, absent abdominal reflexes, and plastic rigidity of the arm and leg; muscular spasms affecting mainly the left side of the body recurred at intervals of approximately 10 seconds. She was restless, uncooperative, and incontinent. The cerebrospinal fluid protein was $56 \mathrm{mg}$. $100 \mathrm{ml}$., cells 4/c.mm., colloidal gold 55,554,211, W.R. negative. Electroencephalography showed a very slow dominant 
rhythm with high voltage bi-phasic complexes recurring approximately every 10 seconds and associated with her muscular spasms. She was subsequently investigated in 1956 in the National Hospital, Queen Square, by Dr. E. Arnold Carmichael. By this time she was completely helpless, took no interest in her surroundings, and lay in an attitude of flexion with little movement apart from occasional slow clenching of the right hand. Repeated EEG recordings were very similar to that of 18 months before: the complexes now recurred at intervals of between $3 \cdot 5$ and 7 seconds against a background that showed very little, if any, rhythmic activity. On ventriculography some dilatation of both lateral ventricles was evident but no other abnormality. Frontal lobe cortical biopsy sections were reported as showing, "considerable gliosis and slight fat granule cell formation in the white matter. The appearances are either those of van Bogaert's subacute sclerosing leucoencephalitis or of Dawson's inclusion body encephalitis, but in the sections so far examined, inclusion bodies have not been found. Active neuronal disintegration is however visible.' She continued to deteriorate very slowly and was ultimately admitted to a mental hospital where she died in July 1958. Necropsy was not performed.

Case 3. A boy aged 8 years was admitted on May 14, 1957. Four weeks previously he had an episode of compulsive coughing and spitting lasting for several days. Thereafter his speech became slurred, his writing deteriorated, he became generally clumsy and began to have spasmodic jerks of his right arm and leg. On examination he was a rather euphoric child whose speech was virtually unintelligible. There was general rigidity of the right arm and leg with spasmodic jerks recurring continually at 10-second intervals. Examination was otherwise negative. The cerebrospinal fluid protein was $60 \mathrm{mg}$. $/ 100 \mathrm{ml}$., cells 2 c.mm., colloidal gold 22,554,330. Electroencephalography showed generalized very high voltage slow wave complexes recurring every 7 to 10 seconds on a background of moderately high voltage 1 to 2 per second waves. A three weeks' course of prednisolone produced no obvious effect, and slow but steady mental and physical deterioration followed. Generalized muscular rigidity developed, and the muscular spasms became less constant though they could always be evoked by any stimulus. He died during an attack of bronchopneumonia on January 2, 1959.

NECROPSY. The necropsy was done by Dr. A. W. Branwood, who has kindly given us permission to report his findings and to examine histological sections of the brain. Apart from the presence of severe confluent bronchopneumonia, significant pathological changes were confined to the central nervous system. The meninges were normal and the brain, which weighed $1100 \mathrm{~g}$. . showed only moderate congestion on macroscopic examination.

Histological Examination. The features are those of a subacute encephalitis affecting mainly the cerebral hemispheres. Here perivascular cellular infiltration is conspicuous (Fig. 1) and occurs in both the tunica adventitia and Virchow-Robin spaces. The infiltrate consists mainly of small round cells of lymphocytic type, but plasma cells, compound granular corpuscles, and scattered larger mononuclear cells are also recognized. and in some foci there are macrophages containing haemosiderin. This perivascular cuffing is seen especially in the white matter but also involves cortical and nuclear grey matter. In the cortex there is neuronal depletion of unselective distribution accompanied by astrocytic and microglial proliferation. Degenerative changes are commonly seen in surviving nerve cells, sometimes with satellitosis, and there are scattered neuronophagic nodules.

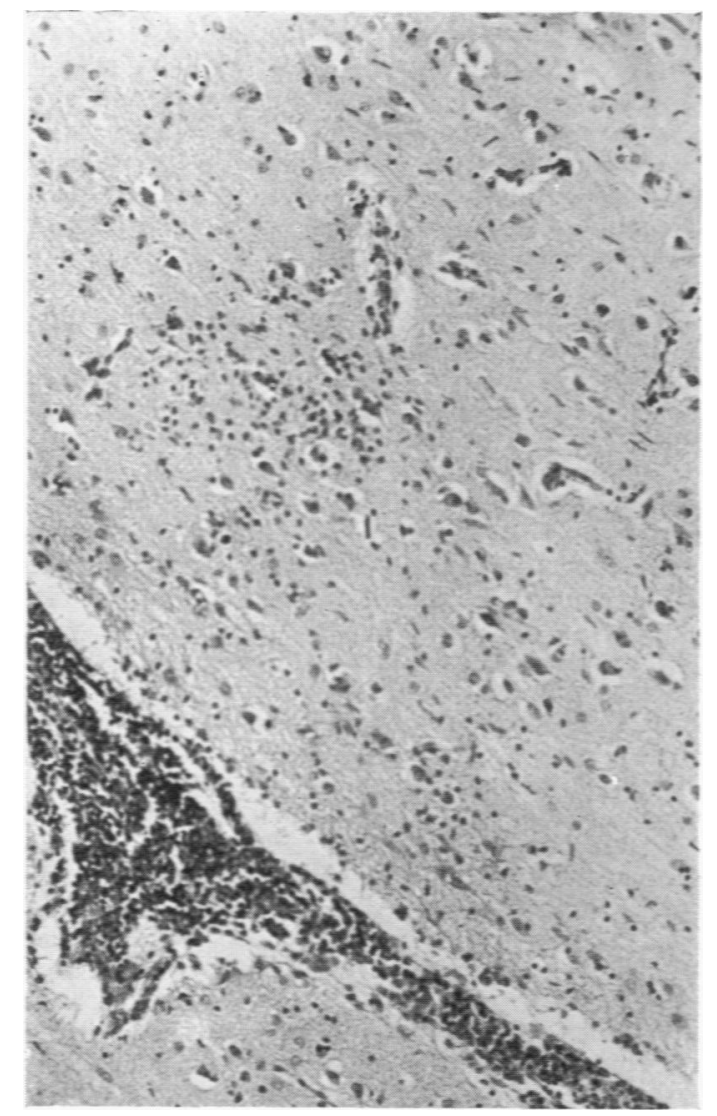

FIG. 1.-Case 3: a field from the cerebral hemisphere showing perivascular cuffing, astrocytic and microglial proliferation with formation of 'rod' cells. (Haematoxylin and eosin. $\times 140$ ).

Microglial rod cells are conspicuous in the cerebral cortex and are present to a lesser extent in the white matter (Fig. 1). Similar inflammatory and neuronal degenerative changes occur in the basal ganglia but are less severe. Irregularly distributed areas of pallor are noted in the subcortical white matter in sections stained to demonstrate myelin. Occasional compound granular cells can be 
identified in these partially demyelinated areas, and there is a marked reactive astrocytic proliferation accompanied by fibrillary gliosis. There are a few foci of cellular infiltration in the leptomeninges. mainly lymphocytic but with some plasma cells.

Mild inflammatory changes are seen in the brain-stem. Perivascular cellular infiltration is much less conspicuous than in the cerebral hemispheres and is often restricted to the tunica adventitia. Occasional nerve cells in the nuclei pontis exhibit chromatolysis or satellitosis. Glial stars are rarely encountered. There is no evidence of demyelination or of astrocytic proliferation.

In the cerebellum pathological features are minimal. There are very scanty perivascular cuffs, occasional foci of astrocytic and microglial proliferation in the white matter, and a very few neuronophagic nodules in the nucleus dentatus. Mild chromatolysis in Purkinje cells is probably attributable to terminal anoxia. There is no recognizable demyelination.

Inclusion bodies were not identified during a prolonged search of the several available sections stained not only by the routine methods but also by Lendrum's phloxinetartrazine technique.

Case 4. A boy aged 8 years was admitted on October 17, 1958, with a three-week history of lethargy and deterioration of school work. One week before admission his right arm and leg began to jerk, he stumbled frequently, and he was unable to control his right arm. On examination he was conscious and co-operative though rather dull mentally. Regularly repeated myoclonic jerks affected the right arm and leg which showed 'lead-pipe' rigidity. Examination was otherwise negative. The cerebrospinal fluid protein was $30 \mathrm{mg}$. $/ 100 \mathrm{ml}$.; cells 2 'c.mm., colloidal gold $00,000,000$. The blood W.R. was negative. Electroencephalography showed generalized very high voltage mixed spike and wave discharges appearing at intervals of 10 to 20 seconds in association with muscular spasms: there was also fairly persistent moderate voltage $2-3 c / s$. slow activity over the left centro-temporal area. Within 10 days of admission speech ceased, he lost all contact with his surroundings, and the myoclonic jerks became more violent. A course of prednisolone lasting two weeks was given without effect. Frequent major and minor fits then appeared and proved completely resistant to a variety of anticonvulsant drugs. A trial of troxidone was followed after 10 days by the rapid development of ulcerative lesions of the mouth and an exfoliative dermatitis which, with an associated pneumonia, led to his death on December 6, 1958.

NeCropsy. This was done by Dr. A. J. Carr who has kindly allowed us to report his findings and to see the histological sections of the brain and other tissues. There was gross exfoliative dermatitis especially over the face, forehead, neck, and ears. Extensive confluent bronchopneumonia was present and was presumably the immediate cause of death. All other significant pathological findings were confined to the central nervous system. The brain weighed $1425 \mathrm{~g}$. and showed intense congestion of vessels in the leptomeninges. Flattening of gyri and narrowing of sulci indicated the presence of increased intracranial pressure, and this appeared to be due to cerebral oedema. The brain and spinal cord were sectioned after formalin fixation, but the only macroscopic abnormality noted was widespread vascular congestion. Specimens were taken from the brain at the time of necropsy for virological examination but virus was not isolated.

Histological Examination. The changes in this case are much less severe than in Case 3 , so much so that they could easily be missed altogether on a cursory examination. Inflammatory lesions can be most easily recognized in the cerebra! hemispheres where there is scattered perivascular infiltration almost entirely by small round cells of lymphocytic appearance, usually confined to the connective tissue of the tunica adventitia, although occasionally the Virchow-Robin space is filled. This perivascular cuffing occurs mainly in the subcortical white matter but can also be identified in the cortex. There is mild neuronal depletion in the cortex and many nerve cells exhibit degenerative changes, occasionally with satellitosis. Astrocytic and microglial increase is noted in the cortex, especially in the parietal and occipital lobes, and the microglial nuclei are often rod-shaped. Very occasional glial stars are present. The basal ganglia in the sections available for examination show negligible changes. In some parts of the white matter slight pallor of myelin staining is noted, but this appears to be due to oedema; compound granular cells are not identified and there is no evidence of gliosis. The meninges for the most part appear normal but in a few fields there is a scanty round cell infiltrate.

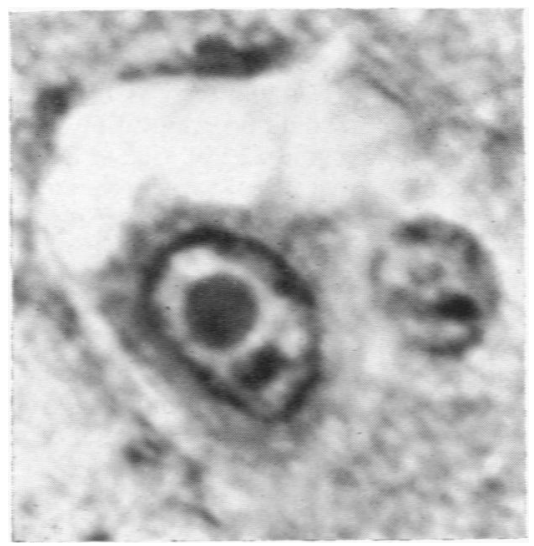

FIG. 2.-Case 4: a nerve cell showing an intranuclear inclusion body which displaces the nucleolus and is surrounded by a clear halo. (Haematoxylin and eosin. $\times 1900$.)

In the bruin-stem there are occasional degenerate nerve cells, some showing satellitosis, and scanty round cell infiltration can be seen in the adventitia of a few vessels. Glial stars are rarely encountered in the pons and medulla. There is no evidence of demyelination. The cerebellum and spinal cord show no notable abnormalities.

Type A eosinophilic intranuclear inclusion bodies are identified in occasional neurones (Fig. 2) and glial cells. 
They are very scanty, concentrated in a few small fields in the cerebral cortex, but more widely dispersed in the pons. Nerve cells are mainly affected, but some of the bodies are found in astrocytic nuclei. Intracytoplasmic inclusions are even more difficult to find, but one of the neurones in the pons seen to contain an intranuclear inclusion also show's irregular cytcplasmic acidophil bodies. Some of the cells with inclusions exhibit degenerative changes whereas others appear to be little affected, and the inclusions are mostly seen in areas where the inflammatory reaction is relatively inconspicuous. Tissues from many other parts of the body, including the alimentary tract, were examined for inclusion bodies but none were found.

Case 5. A young man aged 19 years, older brother of Case 1, was admitted to the Royal Mental Hospital, Aberdeen on August 13, 1959. Four older and two younger sibs were healthy: Case 1 had died at home four years previously, as described above, and another sib had died nine years previously at the age of 1 year and 9 months from tuberculous meningitis. So far as the parents know, there have been no other cases of neurological disease among their relations. Three months before admission the patient had fallen off a wall, striking his head: a superficial wound required three stitches but there was no disturbance of consciousness. From that time his behaviour became peculiar. He seemed 'far away', and on several occasions wandered away on his own and when found and brought home could offer no explanation for his absence. He appeared quite unconcerned by his admission to hospital, though he did not complain of any symptoms. His I.Q. was assessed at 75 , and reading and writing were at the level of a child of 7-8 years. Subsequent inquiry at his old school confirmed that this represented a considerable mental deterioration. The cerebrospinal fluid protein was $50 \mathrm{mg}$. $100 \mathrm{ml}$., cells $4 /$ c.mm., W.R. negative, and colloidal gold 55,531,000. Generalized high voltage slow wave complexes recurred at 7 to 10 second intervals throughout his EEG recording. After a brief period of restless disturbed behaviour he lapsed into a quiet negativistic state in which it became increasingly difficult to make contact with him. Regularly recurring muscle spasms developed, and in the terminal stages he showed an external ophthalmoplegia and increasing generalized rigidity. He developed bronchopneumonia and died on October 14, 1959.

NeCROPSY. Apart from the presence of extensive haemorrhagic bronchopneumonia, significant pathological findings were confined to the central nervous system. The brain weighed $1500 \mathrm{~g}$. and showed no macroscopic abnormalities eitber externally or on transection after formalin fixation.

Histological Examination. The features are very similar to those of Case 3 but less severe, with a widespread subacute inflammatory process affecting principally the cerebral hemispheres. Pallor of myelin staining is less conspicuous than in Case 3 and occurs only in isolated patches, usually subcortical. Astrocytic proliferation and fibrillary gliosis are, however, well established, and there is notable depletion of oligodendroglia in these

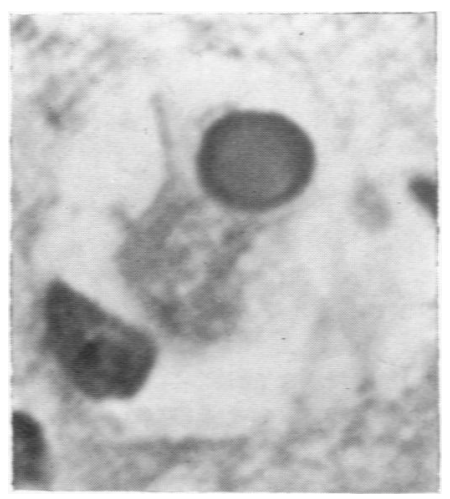

Fig. 3.-Case 5: an inclusion body filling the nucleus of a perineuronal oligodendroglial cell. The body is eosinophilic and the nuclear chromatin is compressed around its margins without an intervening clear halo. The outline of the nucleus of the nerve cell can be faintly seen. (Haematoxylin and eosin. $\times 1700$.)

areas. Lesions in the upper brain-stem are more obvious and more numerous than in Case 3 . There are very scanty changes in the medulla and cerebellum, and the spinal cord shows no significant abnormalities.

Type A intranuclear inclusions are present, but are scanty, and they occur mainly in oligodendroglia although some are seen in nerve cells. The affected oligodendroglial nucleus is usually swollen and almost completely filled by the inclusion body which is surrounded by a dense rim of chromatin without an intervening clear halo (Fig. 3). Small inclusion bodies which do not fill the nucleus are rarely encountered. Similar, spherical or

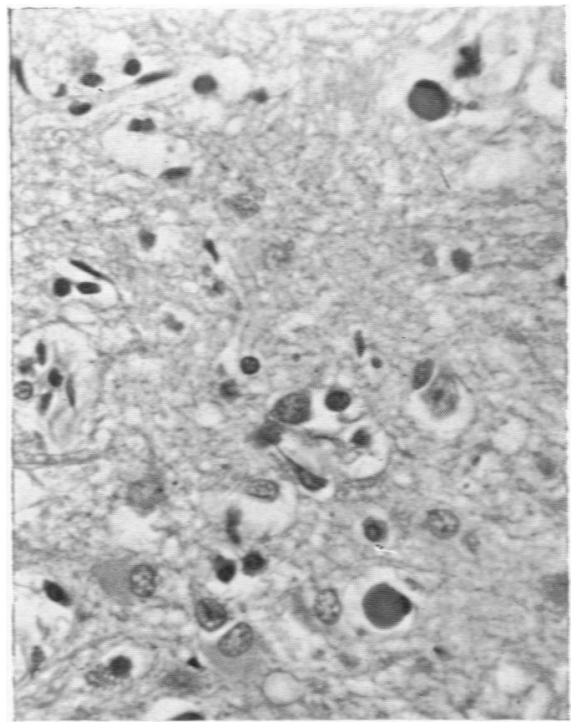

FIG 4.-Case 5: eosinophilic bodies similar to that in the oligodendroglial nucleus in Fig. 3, lying free but with a small crescentic nucleus at the margin. Astrocytic proliferation can also be seen. (Haematoxylin and eosin. $\times 370$. .) 
ovoid, eosinophilic phloxinophilic bodies are also found free in the nervous tissue or in perivascular spaces, and there is sometimes a crescentic nucleus closely apposed to the margin (Fig. 4), possibly that of a microglial cell which has phagocytosed the body. Intracytoplasmic inclusions are not identified.

\section{Discussion}

Incidence. The clinical and electroencephalographic picture presented by this condition is striking and distinctive. It is not a disease which is ever likely to kill before the patient is admitted to hospital. All previous reports are agreed that the onset is invariably in childhood or adolescence. We believe, therefore, that we can claim with some confidence that only 4 cases (Cases $1,3,4$, and 5) of subacute leucoencephalitis have occurred in the NorthEastern Hospital Region of Scotland in the ten-year period, 1952-61. We can say with certainty that no other children with a picture even remotely resembling this have been admitted during this time either to the Royal Aberdeen Hospital for Sick Children or to the children's unit in Aberdeen City Hospital for Infectious Diseases. There are no other children's units in the Region. Case 5 is the only case recorded at either of the two principal mental hospitals serving the Region. The possibility that an adolescent case may have been admitted to an adult medical ward cannot be completely excluded, but we have been unable to trace any such case. The annual incidence in this part of the country would, therefore, appear to be rather less than one per million total population.

During this ten-year period $\mathbf{5 0}$ cases of encephalitis of all types were seen in the children's units in Aberdeen. Of these, 16 were examples of post-infection or post-inoculation encephalitis, 5 were certainly due to virus infection, 4 were Cases $1-4$ here described, and the remainder were of uncertain type though some of these may also have been due to virus infection.

The occurrence of two examples of such a rare condition in one family is of particular interest but uncertain significance. The scarcity of previous reports of familial cases suggests that it is most unlikely that the disease is of purely genetic origin. The interval of over three and a half years between the death of Case 1 and the onset of symptoms in her brother suggests that, if family infection with a rare specific virus is the aetiological factor, the virus must be able to lie dormant for long periods. In the two brothers reported by Lóránd et al. (1962), there was an interval of approximately one year between the death of the first boy and the onset of symptoms in his brother. Alternatively, of course, it may be, as Simpson (1961) suggests, that a common virus such as the hepatitis virus, can produce this uncommon picture in certain rare individuals when acting in conjunction with some other factor. In none of our cases, however, is there a past history of infective hepatitis or of any virus infection other than the common infectious diseases of childhood. If Simpson's theory is correct, the occurrence of two cases in one family suggests that the other factor may be genetically determined. Further speculation is unlikely to be profitable unless more familial cases come to light.

Clinical Features. Van Bogaert (1961) has emphasized that mental deterioration and personality changes are the earliest features of this disease and frequently attract attention before any evidence of organic disease can be detected. Such changes in a previously normal child or adolescent should always arouse suspicion of the onset of this type of encephalitis. It is notable in the present series that Case 5 was admitted to a mental hospital and that Cases 1 and 2 had both been referred for psychiatric opinion some time before their admission for physical investigation. Case 1 had also been examined in the ophthalmic department because of her reading difficulties. Only Cases 3 and 4, whose early evolution was unusually rapid, were sent to hospital because of suspected organic cerebral disease.

The symptoms and course of our cases conformed to the classical picture of this disease of which there are a number o. comprehensive accounts (Foley and Williams, 1953; van Bogaert, 1961; Lóránd et al., 1962). The essential features may be briefly summarized as mental deterioration and impairment of voluntary muscle control progressing inexorably to a final state of complete dementia and helplessness; increasing generalized muscular rigidity, frequently of 'lead-pipe' type; and a wide variety of involuntary movements of which the most frequent and characteristic are myoclonic jerks recurring rhythmically a: intervals of 10 to 20 seconds over periods of weeks or months and tending to disappear in the terminal stages of the disease. Signs of pyramidal tract involvement are as a rule scanty. The total duration of the illness in our cases varied from a maximum of nearly five years in Case 2 to a minimum of two and a half months in Case 4, whose death was probably hastened by his reaction to troxidone administration. Previous reports have shown a similar wide variation in the rate of progress of the disease.

The "paretic' type of colloidal gold reaction in the cerebrospinal fluid is a feature of most reported cases. In Case 4, the only one of our cases to show a negative reaction to this test, the cerebrospinal 


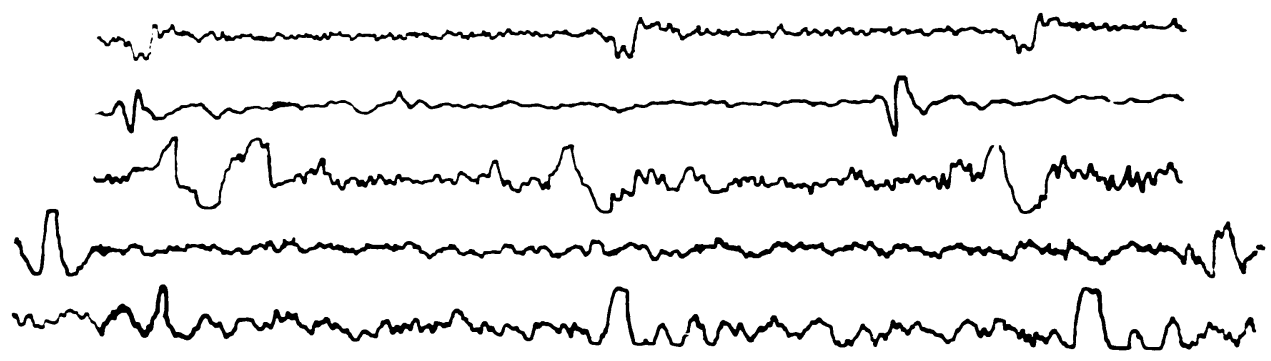

FIG. 5. Sample lead from electroencephalogram of each of the five patients showing in each case regularly recurring high voltage slow wave complexes.

fluid examination was performed three weeks after the onset of symptoms, and it may be that, had the test been repeated later, a positive result would have been obtained. In one of the cases of Foley and Williams (1953), however, a positive reaction was found approximately three weeks from the onset, and the colloidal gold reaction would appear to be a useful test to provide supporting evidence for the diagnosis, even in the early stages of this disease. There is as a rule no increase in cells in the cerebrospinal fluid and the total protein content is normal or slightly raised.

The electroencephalographic records from all 5 cases (Fig. 5) show the highly characteristic generalized, high voltage, bi-phasic, slow wave complexes recurring rhythmically at intervals varying between $3 \cdot 5$ and 20 seconds. The background activity varies from case to case, but as a rule the complexes stand out from it so clearly, in some leads at any rate, that the picture is immediately obvious even to the amateur reader of the electroencephalogram. Lóránd et al. (1962), on the basis of a study of 152 recordings from 31 patients with this disease, consider the repetitive high voltage complexes absolutely pathognomonic and found no similar pattern in a collection of 9,000 electroencephalographic recordings from patients with other illnesses. Where rhythmical myoclonic jerks are present, they are synchronous with the high voltage electroencephalographic complexes, but the latter persist in the absence of any obvious muscular activity. The relation of these two phenomena and the reason for their remarkable periodicity remain quite obscure.

All previous reports are agreed that no form of treatment yet employed has the slightest influence on the course of this disease. Prednisolone was administered to Cases 3 and 4 of the present series with no obvious effect. Van Bogaert (1961) has subsequently expressed the opinion that in some cases cortisone may even accelerate the fatal outcome.
Pathology. The pathological picture in Cases 3, 4, and 5 is that of a subacute encephalitis, and corresponds to previous descriptions of subacute sclerosing (inclusion body) encephalitis. The degree of histopathological change varied in the three cases, and was particularly mild in Case 4, probably because death occurred relatively early in the course of the disease, apparently as a result of a drug sensitivity reaction.

Inclusion bodies were not identified in Case 3 or in the cortical biopsy specimen taken from Case 2, but they were present in Cases 4 and 5 . The difference in distribution of inclusions, however, is of interest in these two brains. In Case 4 they were mainly in nerve cells, with a few in astrocytes, whereas in Case 5 the majority were in oligodendroglia and only a few in nerve cells. The reason for this difference is obscure, and indeed the significance of the inclusions is far from clear. The fact that they often occur in cells apparently unaffected by their presence, and that their distribution in the brain does not correlate with the intensity of the degenerative or inflammatory changes, tends to suggest that their presence may not have aetiological significance.

In view of the discovery of inclusion bodies in ganglion cells of the rectum in a case of inclusion body encephalitis reported by Bodian and Lake (1963), sections of other viscera from Cases 3, 4, and 5 were re-examined, but only in Case 4 had blocks of tissue for histological examination been taken from the alimentary tract. Inclusion bodies were not, however, found in any of these sections.

\section{Summary}

Five cases of subacute sclerosing (inclusion body) encephalitis have been diagnosed in the NorthEastern Region of Scotland in the period 1952-61. The annual incidence of the disease in this area appears to be less than 1 per million total population. Two of these cases were brother and sister. The 
occurrence of this condition in two sibs has only once previously been reported.

The clinical, electroencephalographic, and pathological features of these cases are described.

Attention is drawn in particular to the tendency of this disease to declare itself by personality changes which may be obvious for months before abnormal physical signs appear.

Of the 5 cases, 3 came to necropsy, and 2 of them showed typical inclusion bodies. It is suggested that these inclusions are probably not of aetiological significance.

We wish to express our thanks to Dr. E. Arnold Carmichael and the Medical Committee of the National Hospital, Queen Square, for permission to quote from their records of Case 2, and to Dr. A. M. Wyllie, physician superintendent, Royal Mental Hospital, Aberdeen, for permission to report Case 5. We are also indebted to Professor John Craig for advice and encouragement, and to Professor A. R. Currie for reading the manuscript.

\section{REFERENCES}

Bodian, M. and Lake, B. D. (1963). The rectal approach to neuropathology. Brit. J. Surg., 50, 702.

Dawson, J. R. (1933). Cellular inclusions in cerebral lesions of lethargic encephalitis. Amer. J. Path., 9, 7.

(1934). Cellular inclusions in cerebral lesions of epidemic encephalitis. Arch. Neurol. Psychiat. (Chic.)., 31, 685.

Foley, J. and Williams, D. (1953). Inclusion encephalitis and its relation to subacute sclerosing leucoencephalitis. Quart.J. Med., 46 (n.s. 22), 157.

Lóránd, B., Nagy, T. and Tariska, S. (1962). Subacute progressive panencephalitis. Wld Neurol., 3, 376.

Poser, C. M. and Radermecker, J. (1957). Subacute sclerosing leucoencephalitis. J. Pediat., 50, 408.

Simpson, J. A. (1961). Subacute inclusion-body encephalitis: a possible association with infective hepatitis. Lancet, 2, 685.

van Bogaert, L. (1945). Une leuco-encéphalite sclérosante subaigue. J. Neurol. Neurosurg. Psychiat., 8, 101.

— (1961). The subacute encephalitides. Cerebr. Palsy Bull., 3, 17. 